\title{
ultramicroscopy
}

ELSEVIER

Ultramicroscopy 78 (1999) 175-183

\section{Calculation of near edge structure}

\author{
Peter Rez ${ }^{\mathrm{a}, *}$, Jose R. Alvarez ${ }^{\mathrm{a}, 1}$, Chris Pickard ${ }^{\mathrm{b}}$ \\ ${ }^{a}$ Department of Physics and Astronomy and Center for Solid State Science, Arizona State University, Tempe, AZ 85287-1504, USA \\ ${ }^{\mathrm{b}}$ Cavendish Laboratory, Madingley Road, Cambridge CB3 OHE, UK
}

Received 11 September 1998; received in revised form 4 December 1998

\begin{abstract}
Near edge fine structure has the potential to solve problems related to localised electronic states and bonding. Theory and calculation provide the link between electronic or structural properties and features observed in an electron loss spectrum. A hierarchy of approximations for the calculation of near edge structure features is introduced and the importance of using a self-consistent charge density and potential is emphasised. The use of various electronic structure calculation methods and their application to near edge structure calculation is reviewed. Finally, core hole effects are discussed and examples presented for cubic BN showing that the core hole mainly enhances intensity near threshold. (C) 1999 Elsevier Science B.V. All rights reserved.
\end{abstract}

\section{Introduction}

Using high-resolution spectrometers and imaging filters attached to field emission microscopes it is now a routine matter to record spectra at a resolution of $1 \mathrm{eV}$. All regions of the energy loss spectrum show interesting new features at this level of resolution. The fine structure features just above the thresholds of inner shell edges are of particular interest as they relate to a particular element in a given environment. Materials scientists and other microscope users all would like to make use of this

\footnotetext{
*Corresponding author. Tel.: +1-602-9656449; fax: +1-6029659004.

${ }^{1}$ Current address: Atomic Simulations Group, Department of Mathematics and Physics, Queens Unversity of Belfast Belfast, BT7 1NN, Northern Ireland.

E-mail address: peter.rez@asu.edu (P. Rez)
}

rich extra structure to answer significant microstructural questions. Although progress can be made by comparison of spectra from the same edges in different compounds, the full potential of fine structure analysis can only be realised from a theoretical understanding of the origins of the various features. The theory can then be used as a basis for calculation of fine structure features to be compared with experiment. Near edge structure can often be related to the local environment of the excited atom and can be used to deduce interatomic bond lengths and coordination. Of more interest is the use of near edge structure as a probe of changes in the electronic states as manifested in the conduction band.

In the review for the last conference [1] in this series a hierarchy of approximations in the development of the theory of near edge structure was presented. This hierarchy (shown in revised form as Table 1) provides a framework for the discussion of 
Table 1

Hierarchy of approximations for near edge structure calculations

(1) Atomic theory

Basic edge shapes such as delayed maxima

Atomic multiplet theory gives 1st row transition

$\mathrm{L}_{3}$ and $\mathrm{L}_{2}$ or rare earth $\mathrm{M}_{5}$ and $\mathrm{M}_{4}$ structures

(2) Superposition of atomic potentials or wave functions

Superposition of potentials

Superposition of wave functions

Greens function methods

Linear combination of atomic orbitals

EXAFS single scattering

(LCAO) good for molecular orbital

XANES, old FEFF multiple scattering

features on threshold

(3) Electronic structure calculations-self consistent potentials

Muffin tin methods

Pseudopotential methods

Greens function methods KKR + variants

Augmentation

APW, LAPW, FLAPW LMTO, ASW

Plane wave pseudopotential

(need to reconstruct all e-wave function)

Pseudo-atomic orbital

(4) Core-hole effects

$\mathrm{Z}+1$

Perturbative Clogston-Wolff

Supercell

subsequent developments in the calculation of near edge structure for a number of different systems. In particular, methods suitable for calculating near edge structure changes for systems of importance in materials science such as grain boundaries will be reviewed. The increase in computing power has also made it possible to perform more realistic calculations of the effects of the core hole on the excitation process.

\section{Theory}

Since the probability of an electron passing through a TEM specimen loosing sufficient energy to excite an inner shell electron is quite low, it is sufficient to treat the scattering of fast electrons by first-order perturbation theory and to make use of the general expression for the differential cross section given by [2]

$$
\frac{\mathrm{d}^{2} I}{\mathrm{~d} \Omega \mathrm{d} E}=\frac{4 \gamma^{2}}{a_{0}^{2}}\left|\frac{\langle f \mid \exp (\mathrm{iq} \cdot \boldsymbol{r}) i\rangle}{q^{2}}\right|^{2},
$$

where $q$ is the wave vector, $\gamma$ is a relativistic correction and $\mid \mathrm{i}>$ and $<\mathrm{f} \mid$ are the initial and final states, respectively. For simplicity the effects of dynamical diffraction of the fast electron will be neglected and the fast electrons are treated as plane wave. In practice for inner shell excitations up to about $1.5 \mathrm{kV}$, the characteristic scattering angle $\theta_{\mathrm{E}}$ is much less than the typical Bragg angle, which means that only elastic scattering can move the electron into a different Brillouin zone. This means that the same energy loss spectrum will be observed using an aperture around any Bragg spot. At higher energies inelastic umklapp processes could be significant, though it is doubtful that they would result in changes in the spectrum on the scale of near edge structure. The full theory incorporating all these effects has been discussed by Saldin and Rez [3] and Weickenmieir and Kohl [4] and will not be considered further in this paper.

In most cases, in electron microscopy one can assume that $\boldsymbol{q} \cdot \boldsymbol{r}<1$ and the exponential can be expanded to the first order [5]. The initial state wave functions are confined to the atomic site of the excitation, so it is convenient to use wave functions which are products of radial wave functions and spherical harmonics. The angular integration over the spherical harmonics gives the dipole selection rules for the angular momentum quantum number 
$1^{\prime}=1 \pm 1$. It is then convenient to write the differential cross section as

$$
\frac{\mathrm{d}^{2} I}{\mathrm{~d} \Omega \mathrm{d} E}=\frac{4 \gamma^{2}}{a_{0}^{2} q^{2}}\left[\left|m_{L+1}\right|^{2} \rho_{L-1}(E)+\left|m_{L-1}\right|^{2} \rho_{L-1}(E)\right],
$$

where $m_{1}$ is the matrix element for transitions to final state with angular momentum 1 , and $\rho_{l}$ is the density of states for that particular final state. The dipole approximation should be acceptable for scattering up to $2 \AA^{-1}$ which corresponds to an angle of about $8 \mathrm{mrad}$ at $200 \mathrm{kV}$. Since the square of the matrix element in Eq. (1) is divided by $\mathrm{q}^{4}$, the contribution of the higher order non-dipole terms is considerably suppressed and the dipole approximation is quite acceptable for apertures up to 20-30 mrad at 200-300 kV. Non-dipole effects are only observed with great difficulty using small displaced apertures.

In most cases the matrix elements vary slowly with energy and so the near edge structure represents the angular momentum resolved density of empty states at the site of the excitation. For example a $\mathrm{K}$ shell excitation probes $\mathrm{p}$ states, and an $\mathrm{L}_{23}$ excitation would probe a combination of $\mathrm{s}$ and $\mathrm{d}$ states, depending on the matrix elements.

The wave function for the initial state is an atomic wave function, and can be generated from any suitable Hartree-Fock program, though it is better to use the same atomic program which generates the charge densities or potentials needed as a starting point in the calculation of the final states.

As stated in the last review the differences between theories are all confined to the unoccupied final state wave function. A summary of the various models for the final state wave function is given as Table 1.

The simplest wave function for the final state is an atomic wave function which takes the form of a outgoing spherical wave. Only the delayed maxima which arise from the centrifugal barrier in the potential are predicted with none of the fine structures due to neighbouring atoms [6]. In fact, the theory is completely given by the matrix element terms of Eq. (2).

In the next level of approximation an attempt is made to take account of the effects of neighbouring atoms. This could be done using techniques such as linear combination of atomic orbitals (LCAO) in a molecular cluster for example [7], though most theories are based on the use of Greens functions to describe propagation of an ejected electron from one atom to the next. The scattering from the atom (ion) cores is then represented by phase shifts. Extended fine structure beyond $20-30 \mathrm{eV}$ above threshold is described by a single reflection from a neighbouring atom, which gives structures of the form of a combination of sine waves. The techniques for analysis of this region are well developed and are reviewed by Teo and Joy [8]. When more scattering pathways are considered the near edge structures can be calculated. This is the basis of the XANES method [9] and the early versions of the FEFF code [10]. The key approximation at this level is that the potential for the ejected electron is simply a superposition of atomic potentials and no attempt has been made to take account of any changes such as charge redistribution that arises from bonding. Calculations based on these methods can therefore give no information on the changes in the near edge structure that arise from electronic structure or bonding. They can still be useful for understanding how peak sizes and positions change for the same atom with different nearest-neighbour coordinations in different environments. As there is an extensive literature on both extended fine structure and XANES we shall not give any examples of such calculations here.

The next level of approximation involves generating a self-consistent potential from a ground state charge density and then using this potential to calculate the excited state wave functions and relevant densities of states. For small molecules a Hartree-Fock approach might be used, whereas for solids it is customary to use density functional theory as developed by Hohenberg and Kohn [11] and Kohn and Sham [12]. Rather than work with a many-electron wave function the Shrodinger equation is solved in terms of the ground state charge density which is expressed as a sum of contributions from orthonormal functions.

$$
n(r)=\sum_{i} \psi_{i}^{*}(r) \psi_{i}(r)
$$


The Shrodinger equation is

$$
\begin{aligned}
& \left(\frac{-\hbar^{2}}{2 m} \nabla^{2}+\sum_{\alpha=1}^{N} \frac{Z_{\alpha} e^{2}}{4 \pi \varepsilon_{0}\left|\boldsymbol{r}-\boldsymbol{R}_{\alpha}\right|}\right. \\
& \left.\quad+\int \frac{e^{2} n\left(r^{\prime}\right)}{4 \pi \varepsilon_{0}\left|\boldsymbol{r}-\boldsymbol{r}^{\prime}\right|} d^{3} r^{\prime}+\frac{\delta E_{X C}[n(r)]}{\delta n(r)}\right) \psi_{i}=\varepsilon_{i} \psi_{i},
\end{aligned}
$$

where $e^{2} n\left(r^{\prime}\right) /\left(4 \pi \varepsilon_{0}\left|\boldsymbol{r}-\boldsymbol{r}^{\prime}\right|\right)$ describes the direct Coulomb energy from electron-electron interactions known as the Hartree energy and $\left\{\delta E_{X C}[n(r)] / \delta n(r)\right\}$ describes the energy from electron exchange and correlation. The many electron problem has been reduced to a set of non-interacting single particle equations. Unoccupied state wave functions may also be calculated in this formalism. It is not intrinsically obvious that these are the wave functions that should be used to calculate the energy loss spectrum as given by Eq. (2). In practice agreement with experiment is enough to justify using the Kohn-Sham eigenfunctions and Godby et al. [13] have shown the difference between the Kohn-Sham eigenvalues and the correct quasi-particle energies can be accounted for a rigid shift of the conduction band, at least for silicon. For the purposes of calculating energy loss spectra this amounts to shifting the origin on the energy axis, which is usually considered to be a legitimate way of achieving better agreement between theory and experiment! However, work on other materials shows that this simple rigid shift is not a universal prescription. The practical calculation still requires some formal expression for the exchange and correlation energy. The simplest thing is to substitute the equivalent value for a free electron gas of the same electron density, the so-called local density approximation (LDA). This works quite well for many systems but is not appropriate where interesting effects arise from spin coupling to the core hole which gives the rich multiplet structure observed in transition metal $\mathrm{L}_{23}$ and rare earth $\mathrm{M}_{45}$ edges, or where spin coupling between neighbouring atoms is important, as for example in the theoretical description of magnetism.

Having settled on the Kohn-Sham equations under the local density approximation it is then only a matter of deciding on which technique should be used to solve them. All methods rely on using Blochs theorem which states that the wave function in a periodic solid has the form

$\psi_{k}(\boldsymbol{r})=\exp (\mathrm{i} \boldsymbol{k} \cdot \boldsymbol{r}) u_{k}(\boldsymbol{r})$.

The initial atomic charge densities (taken from a Hartree-Fock program such as Herman Skillman [14]) are combined to form an initial estimate of the charge density. An iterative scheme is constructed in which the occupied wave functions are used to calculate a new charge density which is mixed with the previous charge density for use in the next iteration. Convergence is assumed when the total energy is minimised. The final ground state charge density is used to calculate the energy bands and wave functions for the unoccupied states. To accurately reproduce structures in the density of states the calculation has to be performed with many more $k$ points than the calculation of the ground state charge density. There is no reason, in principle, why different computer codes might not be used for these two independent steps.

The most convenient methods to use for the calculation of energy loss localised projected densities of states are those that already work with a localised basis such as the muffin tin methods. Spherical muffin tins surround each atom and in the region of the spherical muffin tins the solution is expressed as a product of a radial wave function and spherical harmonics. It is a simple matter to extract the relevant projected density of states at a particular site. One muffin tin method, the KKR method, is very similar to the XANES and EXAFS schemes described above. The region between the muffin tins has a constant potential and Greens functions can be used to describe propagation from one atom to the next. We have found that a variant of this method, the Layered KKR method (LKKR), due to Maclaren and colleagues [15] is particularly useful for investigating near edge spectra, especially in systems with grain boundaries. As the name implies the system is divided into layers. Scattering within the layer is treated by the spherical wave Greens functions as in EXAFS or XANES, scattering between the layers is described using plane waves. It is possibly to solve "exactly" for bulk regions and to build up complicated interfaces with bulk on either side. 
Intensity

(arb. units)

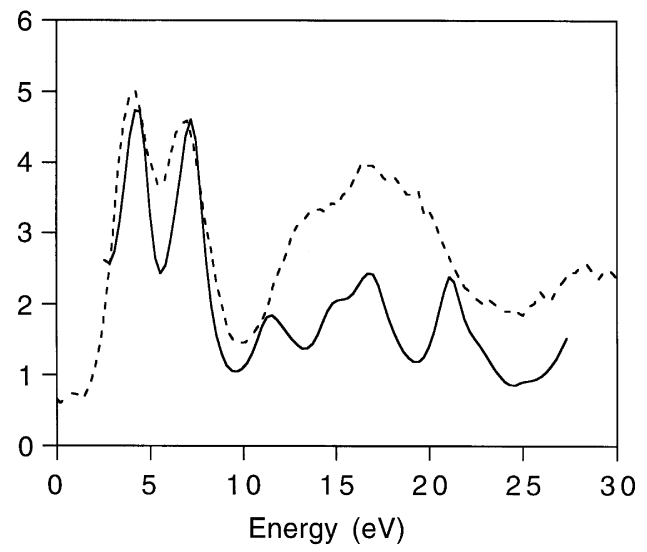

Fig. 1. Comparison of LKKR calculation of oxygen $\mathrm{K}$ edge from the rutile form of $\mathrm{TiO}_{2}$ (solid line) with the experiment (dashed line).

An example of an LKKR calculation for the oxygen $\mathrm{K}$ edge in the rutile form of $\mathrm{TiO}_{2}$ is given as Fig. 1. Note that the calculation shows the molecular orbital $\mathrm{t}_{2 \mathrm{~g}} \mathrm{e}_{\mathrm{g}}$ splitting of about $1 \mathrm{eV}$ at the threshold. This cannot be reproduced by a Greens function multiple scattering calculation that does not use the self-consistent charge density, as shown in our prior publication [1]. Fig. 2 shows the results for the oxygen $\mathrm{K}$ edge in a perovskite material, $\mathrm{SrTiO}_{3}$. There is good agreement between this and the experimental measurements which are not shown in the figure. Other examples are given in a recent publication [16]. As the KKR method is identical to EXAFS and XANES for higher energies above threshold, it should reproduce those features as well as describe the near edge region.

Many workers feel that the main disadvantage with the KKR method is the use of a constant potential between the muffin tins. It is felt that this is inadequate for materials with directional bonds, though the success of the calculation for the oxygen $\mathrm{K}$ edge in rutile would suggest otherwise. The Augmented Plane Wave (APW) method is a muffin tin method where this deficiency can be corrected. The APW method uses plane waves to describe the wave function in the region between the muffin tins. When using a plane wave expansion the interstitial potential can be expressed as a Fourier series which
Intensity

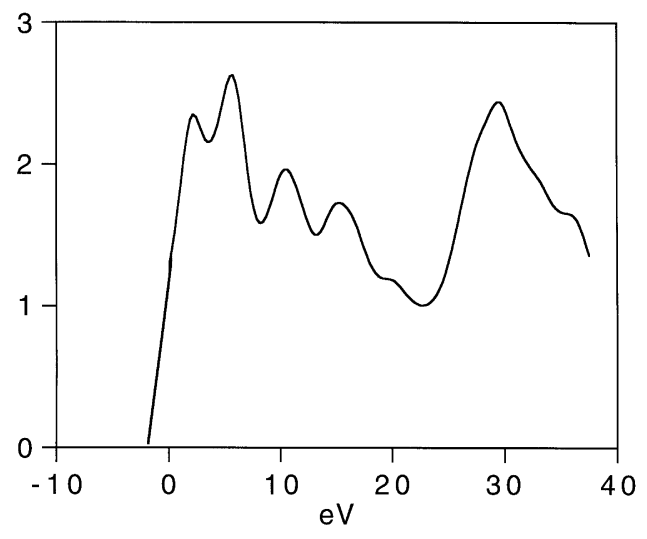

Fig. 2. LKKR calculation of the $\mathrm{O} \mathrm{K}$ edge from strontium titanate.

allows for arbitrary bonding charge distributions. This version of the APW method is known as a full potential APW. The problem with APW for band structure and density of states calculations is that a large matrix must be diagonalised at each energy. This is computationally very expensive and Anderson came up with a simplification in which a combination of the Hamiltonian matrix at one energy and its energy derivatives were used instead [18]. The result is called a linearised APW and a combination of full potential and linearised APW (abbreviated to FLAPW) is available as a standard package [17]. The linearisation scheme was also applied to the muffin tin orbital method proposed by Anderson which is a particularly economical scheme for calculating electronic structures with a minimal basis set [18]. The energy about which derivatives are taken is usually around the centre of the valence band which will give excellent results for total energy and other ground state properties. One must be cautious in applying any linearised scheme to the unoccupied states that form the basis of near edge structure. As Anderson pointed out, the departures from the linear interpolation go as $\left(E-E_{\mathrm{v}}\right)^{4}$, where $E$ is the energy and $E_{\mathrm{v}}$ is the approximate energy for the centre of the valence band. This can result in significant error even between 10 and $20 \mathrm{eV}$ above threshold. LAPW and 


\section{Calculated L2,3 near-edge structures}

LAPW Method

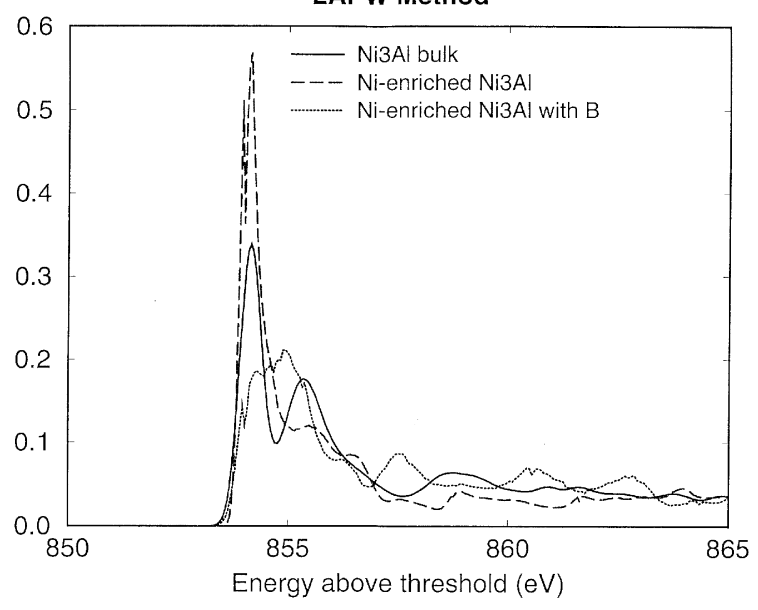

Fig. 3. LAPW calculation of the $\mathrm{Ni} \mathrm{L}_{3}$ edge from bulk $\mathrm{Ni}_{3} \mathrm{Al}$ (solid line), a pure $\mathrm{Ni}$ plane in (dashed line), and $\mathrm{Ni}$ atoms in the $\mathrm{Ni}$ rich plane with interstitial B.

related methods will do well for the detailed shape just above threshold. An example which also shows how calculations can support interpretation of experimental results is given as Fig. 3. FLAPW calculations of $\mathrm{Ni} \mathrm{L}_{3}$ edge are shown for bulk $\mathrm{Ni}_{3} \mathrm{Al}$, at the site of a pure $\mathrm{Ni}$ layer in a $\mathrm{Ni}_{3} \mathrm{Al}$ supercell and also at the site of the same layer when boron is added. Similar results were obtained with the LKKR method. The height of the peak at threshold goes up for pure $\mathrm{Ni}$, but is reduced to the level of the peak from $\mathrm{Ni}$ in bulk $\mathrm{Ni}_{3} \mathrm{Al}$ when Boron is added. These calculations show agreement with the experimental results of Muller [19] who investigated EELS fine structure for boundaries in $\mathrm{Ni}_{3} \mathrm{Al}$ with and without B segregation.

Devising a method that can handle the deep potential wells of the ion cores at the atomic sites and the relatively flat regions between them has always been a problem in the calculation of electronic structure. Plane waves have many advantages in electronic structure calculations, notably the simplicity of the theory and any computer programs based on that theory. In the ion cores many plane waves would be required to successfully represent the wave function. Separating the ion cores as muffin tins is one solution. Another approach is to replace the ion core potential with another potential, the so-called pseudopotential, made up from the nuclear potential and a contribution from the core electrons. It is assumed that the core does not change when the atom moves from one environment to another. The advantage is that the potential is smoother, the valence electron wave functions, now pseudowavefunctions, can be made nodeless and can be described by a relatively small number of plane wave components. It does not matter that pseudowavefunctions are used when calculating many of the ground state properties such as total energy. It is also relatively straightforward to implement schemes for both ionic and electronic relaxation with plane wave pseudopotential codes allowing for the theoretical explanation of the stability of different crystal structures. Recent developments such as ultra-soft pseudopotentials have extended the range of elements and it is now possible to treat systems with thousands of atoms using modern plane wave codes [20]. For calculating near edge structure we need the local angular momentum projected density of states so it is essential to recreate the true wave function from the pseudowavefuction. Following the approach of van der Walle and Blochl [21] the pseudowavefunction need only be corrected within a sphere of radius $r_{\mathrm{c}}$. The all electron wave function is given by

$|\psi\rangle=|\tilde{\psi}\rangle+\sum_{i}\left(\left|\phi_{i}\right\rangle-\left|\tilde{\phi}_{i}\right\rangle\right)\left\langle\tilde{\phi}_{i} \mid \tilde{\psi}\right\rangle$,

where $\left|\phi_{\mathrm{i}}\right\rangle$ and $\left|\tilde{\phi}_{\mathrm{i}}\right\rangle$ are the partial waves for the full and pseudopotentials, respectively, normalised within $r_{\mathrm{c}}$, and $|\psi\rangle$ and $|\tilde{\psi}\rangle$ are the all-electron wave function and pseudowavefunction

In practice it is easier to directly calculate the dipole matrix elements

$$
\begin{aligned}
\left\langle\phi_{\mathrm{c}}|r| \psi\right\rangle= & \left\langle\phi_{\mathrm{c}}|r| \tilde{\psi}\right\rangle+\sum_{i}\left(\left\langle\phi_{\mathrm{c}}|r| \phi_{i}\right\rangle\right. \\
& \left.-\left\langle\phi_{\mathrm{c}}|r| \tilde{\phi}_{\mathrm{i}}\right\rangle\right)\left\langle\tilde{\phi}_{\mathrm{i}} \mid \tilde{\psi}\right\rangle
\end{aligned}
$$

rather than try to resolve angular momentum components.

Another approach is to use pseudopotentials, but still retain the atomic orbital character of the all electron wave function as a combination of pseudo-atomic orbitals [22]. These are already 
labelled by angular momentum quantum number and the relevant local angular momentum projected density of states can then be calculated directly. This approach has been used in our previous work where near edge structures for diamond, graphite, boron nitride and silicon carbide were published [23]. The major problem with pseudo-atomic orbitals is that they do not form a complete set of states at high energies in the conduction band. The method is therefore unable to give reliable results for the transition between the near edge region and the extended fine structure region, starting at about $10 \mathrm{eV}$ above threshold.

The final level of approximation in the hierarchy is to attempt to take account of the dynamics of the excitation process. It is safe to assume that the time for the excitation is fast compared to the relaxation time for the lattice. It is therefore only necessary to consider the electronic states in the presence of the core hole. In a metal where charge flows freely a core hole will have negligible effect as it will be fully screened. For insulators where screening is less effective conduction band states will be pulled down in energy and the wave function will be contracted. This will lead to a shift towards lower energies and an increase in the size of any threshold peaks. One way to include core hole effects in a calculation is to assume that any local change in potential is perturbative. An example is the Clogston-Wolff method in which the effect of the core hole is parameterised by the depth of the core-hole potential and the degree of hybridisation between orbitals [24]. Another popular approach is to take account of the absence of the core electron by adding one to the nuclear charge, the $Z+1$ approximation. In calculations where a supercell is already being used to investigate near edge structure around defects such as boundaries (an example would be the calculation for the $\mathrm{Ni} \mathrm{L}_{23}$ edge for the grain boundaries described above), then it is no more difficult to replace the atom at the excitation site with an atom with the appropriate core electron missing. The electronic structure calculation is then performed and the appropriate local density of states calculated. With the pseudopotential method this is not entirely a trivial exercise as a new set of pseudopotentials have to be calculated. Examples are given as Fig. 4 for the boron and nitrogen
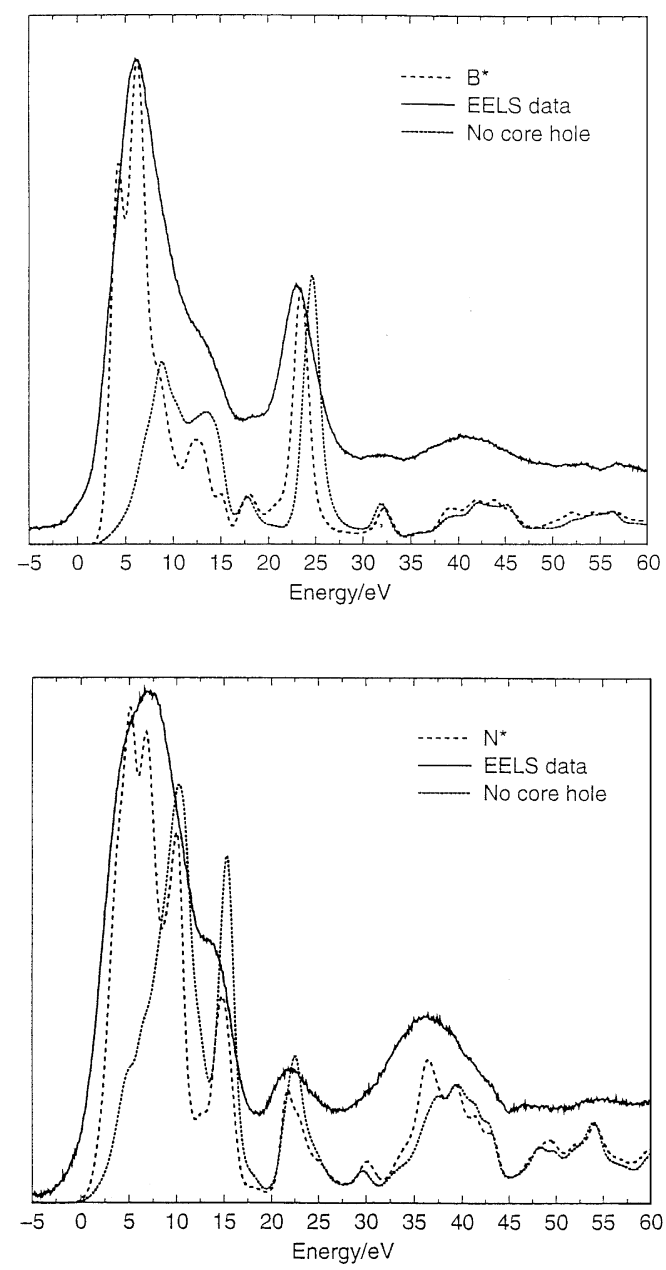

Fig. 4. Plane wave psudopotential calculations for $B$ and $\mathrm{N}$ K edges from cubic BN compared with EELS data. The dotted line shows the results of a calculation without a core hole, the dashed line shows the effects of the core hole.

$\mathrm{K}$ edges in cubic boron nitride. The calculation without the core hole is very similar to the earlier pseudoatomic orbital calculations reported by Weng et al. [23]. As expected the core hole enhances the spectrum peak at threshold. It is interesting to note that this accounts for nearly all the discrepancy between the earlier calculation and experiment in this region. However, comparison with experiment shows that the relative height of the first spectral peak is too large. This suggests that the model overstates core-hole effects and that screening is more important in this system. 


\section{Conclusions}

The detailed calculation of near edge structure attempting to match every feature of experimental measurements is clearly an arduous, maybe even a hopeless, task. We have shown how theory and calculation can aid in the understanding of near edge fine structure, by showing what features arise from a given level of approximation in the theory, and how they change with respect to calculation parameters. The hierarchy of approximations proposed in previous work is shown to still provide a useful framework for the analysis of near edge structures.

The atomic description only provides a picture of the slow variation of edge shape due to delayed maxima. The next level of approximation, where interaction with neighbouring atoms is considered but no attempt made to describe bonding, can only reproduce features related to coordination and interatomic distances. A LCAO approach can give useful qualitative ideas on the near edge region, but is hard to extend into the extended fine structure region or to extract quantitative information. To capture the subtleties of near edge structure that could arise from details in bonding and electronic structure a true self-consistent calculation of ground state charge density and unoccupied electronic states is required. Any of the methods of electronic structure calculation based on Kohn-Sham equations of density functional theory can potentially be used. The method chosen should reflect the nature of the problem and convenience of use. We have found that the LKKR method, which can be formally related to the EXAFS and XANES methods, is particularly well suited to near edge structure calculations in problems involving atoms at boundaries. It can describe fine structures both in the threshold and the extended fine structure region, the main disadvantage being a limitation to spherical muffin-tin potentials. Although this is not serious for metals it could cause problems in materials with highly directional bonds such as semiconductors. An interesting solution to this problem might be to use a Greens function method for the calculation of the near edge structure based on the self-consistent potential from FLAPW. Recent developments of the plane wave pseudopotential method also show promise for calculating the whole range of fine structure in a wide selection of materials.

When all these steps have been taken it might then be necessary to include core-hole effects, which is best done using a supercell. This might involve only marginal extra computational cost if a supercell is already being used, as in a surface or boundary problem. In the examples presented here the main effect of the core hole is to enhance the intensity of the threshold peak and also pull it towards lower energies.

This does not mean that near edge structure calculation is a routine activity, to be compared with high-resolution image simulation. Even when using standard electronic structure calculation programs there are still many issues to be addressed on parameter choice and calculation convergence. Different methods work best for different systems and judicious choices must be made even with a generous allotment of computer time. There are still important systems such as quartz and related silicates where calculations are still not good enough to be useful in explaining the interesting and rich fine structures.

\section{Acknowledgements}

We would like to acknowledge partial support from the NSF through grant DMR 930-6253. We are also grateful to Dr. Mike Payne of the Cavendish Laboratory for his advice and encouragement and to Dr. Laurence Garvie for experimental spectra and discussions.

\section{References}

[1] P. Rez, J. Bruley, P. Brohan, M. Payne, L.A.J. Garvie, Ultramicroscopy 59 (1995) 159.

[2] R.F. Egerton, Electron Energy Loss Spectroscopy in the Electron Microscope, Plenum Press, New York, 1986.

[3] D.K. Saldin, P. Rez, Philos. Mag. 55 (1987) 481.

[4] A. Weickenmeier, H. Kohl, Philos. Mag. 60 (1989) 467.

[5] P. Rez, X. Weng, H. Ma, Microsc. Microanal. Microstruct. 2 (1991) 143.

[6] R.D. Leapman, P. Rez, D.F. Mayers, J. Chem. Phys. 72 (1980) 1232. 
[7] W.M. Skiff, R.W. Carpenter, S.H. Lin, J. Appl. Phys. 58 (1985) 3463.

[8] B.K. Teo, D.C. Joy, EXAFS Spectroscopy, Techniques and Applications, Plenum Press, New York, 1981.

[9] P.J. Durham, J.B. Pendry, C.H. Hodges, Comput. Phys. Commun. 25 (1982) 193.

[10] J. Mustre de Leon, J.J. Rehr, S.I. Zabinsky, R.C. Albers, Phys. Rev. B 44 (1991) 4146.

[11] P. Hohenberg, W. Kohn, Phys. Rev. 136 (1964) B 864.

[12] W. Kohn, L.J. Sham, Phys. Rev. 140 (1965) 1133.

[13] R.W. Godby, M. Schluter, L.J. Sham, Phys. Rev. Lett. 56 (1986) 2415.

[14] F. Herman, S. Skillman, Atomic Structure Calculations, Prentice-Hall, Englewood Cliffs, NJ, 1963.

[15] J.M. Maclaren, S. Crampin, D. Vvedensky, J.B. Pendry, Phys. Rev. B 40 (1989) 12164.

[16] P. Rez, J.M. Maclaren, D.K. Saldin, Phys. Rev. B 57 (1997) 2621
[17] P. Blaha, K. Schwarz, P. Sorantin, S.B. Trickey, Comput. Phys. Commun. 59 (1990) 399.

[18] O.K. Anderson, Phys. Rev. B 12 (1975) 3060.

[19] D.A. Muller, S. Subramanian, P.E. Batson, S.L. Sass, J. Silcox, Phys. Rev. Lett. 75 (1995) 4744.

[20] M.C. Payne, M.P. Teter, D.C. Allan, T.M. Arias, J.D. Joanopoulos, Rev. Mod. Phys. 64 (1992) 1045.

[21] C.G. van de Walle, P.E. Blochl, Phys. Rev. B 47 (1993) 4244.

[22] O.F. Sankey, D.J. Niklewski, Phys. Rev. B 40 (1989) 3979.

[23] X. Weng, P. Rez, O.F. Sankey, Phys. Rev. B 40 (1989) 5694.

[24] P.J.W. Weijs, M.T. Czyzyk, J.F. van Acker, W. Speier, J.B. Goedkoop, H. van Leuken, H.J.M. Hendrix, R.A. de Groot, G. van der Laan, K.H.J. Buschow, G. Wiech, J.C. Fuggle, Phys. Rev. B 41 (1990) 11899. 